(2) Open Access Full Text Article

ORIGINAL RESEARCH

\title{
Analysis of Risk Factors for Poor Short-Term Outcomes in Acute Cardioembolic Stroke Patients without Reperfusion Therapy
}

Yikun Guo'

Min Zhang'

Yan $\mathrm{Su}^{2}$

Jianfang Liu'

Hongran Fu'

Qian Wang'

Yun Chen'

'Department of Neurology, The Affiliated Changzhou No.2 People's Hospital of Nanjing Medical University, Changzhou, Jiangsu Province, People's Republic of China; ${ }^{2}$ Department of Radiology, The Affiliated Changzhou No.2 People's Hospital of Nanjing Medical University, Changzhou, Jiangsu Province, People's Republic of China
Correspondence: Yikun Guo Department of Neurology, The Affiliated Changzhou No.2 People's Hospital of Nanjing Medical University, 68\# Middle Gehu Road, Changzhou, 213000, Jiangsu Province, People's Republic of China

Tel +86-5I9-8I087079

Fax +86-519-810877II

Email guoyikun000@163.com
Purpose: Few clinical indicators of a poor outcome have been defined in acute cardioembolic stroke (CES) patients. We would like to explore practical clinical factors that can predict poor outcomes of CES in the early stage.

Patients and Methods: In this single-center, retrospective, observational study, 251 consecutive patients with acute CES who did not undergo reperfusion therapy were evaluated. On the basis of the modified Rankin Scale (mRS) score at 3 months, patients were divided into the good functional outcome group $(\mathrm{mRS} \leq 2)$ and the poor functional outcome group ( $m R S \leq 3)$. Risk factors were analyzed and the independent indicators for a poor outcome were identified using a binary logistic regression model.

Results: One hundred (39.8\%) patients had a poor outcome. Patients in the poor outcome group were significantly older $(\mathrm{P}=0.002)$ and had significantly higher baseline National Institutes of Health Stroke Scale (NIHSS) score compared with those with a good outcome $(\mathrm{P}<0.001)$. After adjusting for potential confounders, the baseline NIHSS score $(\mathrm{P}<0.001)$, moderate to severe leukoaraiosis $(\mathrm{P}=0.011)$, non-symptomatic intracranial hemorrhage $(\mathrm{P}=$ 0.019), stroke-associated pneumonia $(\mathrm{P}=0.001)$, and fasting glucose $(\mathrm{P}=0.040)$ were independent risk factors for a poor outcome.

Conclusion: The short-term outcome in acute CES patients without reperfusion therapy can be predicted by using five practical clinical factors. These indicators should attract more attention.

Keywords: acute cardioembolic stroke, risk factor, functional outcome, reperfusion therapy, leukoaraiosis

\section{Introduction}

Ischemic stroke is a major cause of death and long-term disability worldwide. ${ }^{1}$ It is a heterogeneous disease, and a main subtype of ischemic stroke is cardioembolic stroke (CES), which accounts for around 15-30\% of all cases and most commonly derives from non-valvular atrial fibrillation. ${ }^{2}$ As the thrombus from the heart suddenly blocks the intracranial artery, patients with CES usually have a sudden onset and large infarction volume which result in a poor outcome. ${ }^{3,4}$ Studies have shown that the incidence of a 6-month unfavorable prognosis (modified Rankin scale, $m R S \geq 3$ ) in these patients can be as high as $66 \%$, and a more severe stroke is the main factor that contributes to a poor prognosis. ${ }^{5,6}$ Recently, the CES patient outcomes have been greatly improved with the increasing use of reperfusion therapies such as intravenous thrombolysis and mechanical thrombectomy, but 
a high percentage of patients may still die or be seriously disabled. ${ }^{7,8}$ In addition, the overall proportion of ischemic stroke patients who receive reperfusion therapy remains low. ${ }^{9,10}$ Therefore, early determination of prognostic factors for CES without reperfusion therapy is of great significance.

Most studies that have focused on the outcome of ischemic stroke have combined patients with various pathologies into one group, which is unlikely to result in conclusive findings. Moreover, few of these studies contain immediately adoptable clinical indicators. In this study, we aimed to explore the risk factors for the 3-month functional outcome of CES patients without reperfusion therapy.

\section{Patients and Methods Subjects}

For this single-center, retrospective, observational study, we retrieved data from the stroke center database of Changzhou No.2 People's Hospital, Jiangsu Province, China. This study was approved by the Clinical Research Ethics Committee of the Affiliated Changzhou No.2 People's Hospital of Nanjing Medical University (approval number: 2018KY032-01; approved on 20 December, 2018 with further agreement to extend the study time). A written informed consent was obtained from the patients or their legally authorized representatives. All patient data were de-identified. This study was conducted in accordance with the Declaration of Helsinki.

Consecutive patients with first-ever acute stroke within 48 hours of onset from June 2014 to September 2019 were included. All patients had known or newly- diagnosed non-valvular atrial fibrillation, and stroke events were confirmed by magnetic resonance imaging (MRI) or computed tomography (CT) and treated in accordance with the corresponding guidelines. Other inclusion criteria were as follows: (1) age 18 to 80 years; (2) anterior circulation infarction; and (3) involvement of at least one side of the middle cerebral artery. Patients were excluded if they had reperfusion therapy, died rapidly after admission (within 2 days), or had no brain MRI/ magnetic resonance angiography (MRA) or CT/ computed tomography angiography (CTA), which is essential to estimate the stroke subtype.

\section{Diagnosis of Cardioembolic Stroke}

Essential tests such as electrocardiogram (ECG), transthoracic echocardiography, cervical vascular ultrasound, and cranial CTA/MRA were routinely performed. Thus, the known or newly- diagnosed non-valvular atrial fibrillation was confirmed, and the CES stroke subtype was defined using the TOAST (Trial of Org 10172 in Acute Stroke Treatment) stroke classification. ${ }^{11}$

\section{Baseline Assessment and Clinical Evaluation}

Baseline demographics, vascular risk factors, and clinical and laboratory characteristics were obtained from medical records. Vascular risk factors including hypertension, diabetes mellitus, hyperlipidemia, congestive heart failure, and smoking were documented. Neurological deficit was measured using the National Institutes of Health Stroke Scale (NIHSS). Leukoaraiosis (LA) was graded using the Fazekas visual scoring scale for the CT or MRI scan. ${ }^{12}$ Mild LA was defined as Fazekas score $\leq 2$, and moderate to severe LA as Fazekas score $\geq 3$.

An intracranial hemorrhage ( $\mathrm{ICH})$ was defined as symptomatic $(\mathrm{sICH})$ if the patient had clinical deterioration causing an increase in the NIHSS score by $\geq 4$ points and as non-symptomatic (nsICH) otherwise. ${ }^{13}$

Stroke-associated pneumonia (SAP) was diagnosed in the presence of fever, purulent sputum, abnormal respiratory examination, pathologic chest X-ray findings, and/or leukocytosis or leukopenia (white blood cells count $\geq$ $10 \times 109$ or $\leq 4 \times 109 / \mathrm{L}$, respectively). ${ }^{14}$

\section{Functional Follow-Up}

Functional outcome was evaluated using the mRS through an outpatient clinic visit or telephone inquiry at 3 months after stroke onset. A doctor and a trained nurse separately judged the functional status of each patient and then achieved a consistent result. On the basis of this judgement, the patients were divided into a good outcome group $(\mathrm{mRS} \leq 2)$ and a poor outcome group $(\mathrm{mRS} \geq 3)$.

\section{Statistical Analysis}

Continuous variables with a normal distribution were presented as the mean \pm standard deviation (SD) and compared using the Student's $t$-test. Continuous variables with non-normal distribution were shown as the median (interquartile range, IQR) and compared using the MannWhitney $U$-test. Categorical variables were expressed as percentage and compared with Pearson's $\chi^{2}$ test or Fisher's exact test. Variables with a two-tailed $P<0.2$ in the univariate analysis were used as candidates for the 
Table I Baseline Characteristics

\begin{tabular}{|l|l|}
\hline Variable & $\mathrm{n}=25 \mathrm{I}$ \\
\hline Age, years, median (IQR) & $73(67,77)$ \\
Male sex, $\mathrm{n}(\%)$ & $138(55)$ \\
Smoking, $\mathrm{n}(\%)$ & $62(24.7)$ \\
Hypertension, $\mathrm{n}(\%)$ & $196(78.1)$ \\
Diabetes mellitus, $\mathrm{n}(\%)$ & $52(20.7)$ \\
Hyperlipidemia, $\mathrm{n}(\%)$ & $43(17.1)$ \\
Congestive heart failure, $\mathrm{n}(\%)$ & $52(20.7)$ \\
Baseline NIHSS score, median (IQR) & $7(3,14)$ \\
Moderate to severe LA, $\mathrm{n}(\%)$ & $63(25.1)$ \\
sICH, $\mathrm{n}$ (\%) & $27(10.8)$ \\
nsICH, $\mathrm{n}$ (\%) & $46(18.3)$ \\
Stroke-associated pneumonia, $\mathrm{n}(\%)$ & $79(3 \mathrm{I} .5)$ \\
Anticoagulant therapy, $\mathrm{n}(\%)$ & $120(47.8)$ \\
Stroke recurrence, $\mathrm{n}(\%)$ & $8(3.2)$ \\
\hline Laboratory findings, mmol/L (mean \pm SD) & \\
\hline Total cholesterol & $4.1 \pm 0.8$ \\
Triglyceride & $1.2 \pm 0.8$ \\
LDL-C & $2.2 \pm 0.7$ \\
Fasting glucose & $6.3 \pm 2.2$ \\
\hline
\end{tabular}

Abbreviations: IQR, interquartile range; NIHSS, National Institutes of Health Stroke Scale; LA, leukoaraiosis; sICH, symptomatic intracerebral hemorrhage; nsICH, non-symptomatic intracerebral hemorrhage; LDL-C, low-density lipoprotein cholesterol; SD, standard deviation.

independent variables that were entered into the binary logistic regression model (Enter method). The results were expressed as the odds ratio (OR) and $95 \%$ confidence intervals (CI). A two-tailed $P<0.05$ was considered to be statistically significant. All statistical analysis was performed on SPSS 22.0 (IBM Corp., Chicago, IL, USA).

\section{Results}

\section{Baseline Information}

Among the 292 patients who initially met the inclusion criteria, 41 patients were lost to follow-up. Thus, 251 patients were included for the final analysis, among whom 138 (55\%) were men. The age range was 40 to 80 years, with a median age of 73 years. The median admission NIHSS score was 7 (IQR 3-14). Approximately 78\% of the subjects had hypertension and $25 \%$ had moderate to severe LA. During hospitalization, 73 (29.1\%) patients developed hemorrhagic transformation, among whom $27(10.8 \%)$ were symptomatic. Additionally, SAP occurred in 79 (31.5\%) patients (Table 1).

\section{Prognosis Distribution}

At 3 months, 19 (7.6\%) patients had died and eight (3.2\%) patients had recurrent stroke. Although the most serious cases were excluded due to rapid death, there was a high frequency of severe disability and death ( $\mathrm{mRS} \geq 4,22 \%)$ in the study population. Even in the good outcome group, up to $19.1 \%$ of the patients had $\mathrm{mRS}=2$, indicating mild neurological dysfunction (Figure 1).

\section{Differences in Risk Factors and Clinical Findings Between the Two Outcome Groups}

There were 151 (60.2\%) patients who were placed into the good functional outcome group and 100 (39.8\%) who were placed into the poor functional outcome group. Compared with patients in the good outcome group, those who had poor outcome were older ( 75 vs 72 years; $\mathrm{P}=0.002)$, had a higher baseline NIHSS score (15 vs 4 ; $\mathrm{P}<0.001$ ), and had more severe LA (moderate to severe LA: $39.0 \%$ vs $15.9 \%, \mathrm{P}<0.001)$. Additionally, patients with a poor outcome had higher fasting glucose level (7.0 \pm 2.7 vs $5.8 \pm 1.7 ; \mathrm{P}<0.001)$ and lower triglyceride level ( $1.0 \pm 0.4$ vs $1.3 \pm 0.9 ; \mathrm{P}=0.005)$ compared with the good outcome group. Patients in the poor outcome group also developed higher rates of $\mathrm{sICH}(17.0 \%$ vs $6.6 \%, \mathrm{P}=$ $0.009)$, $\mathrm{nsICH}(30 \%$ vs $10.6 \%, \mathrm{P}<0.001)$, and SAP $(66.0 \%$ vs $8.6 \%, \mathrm{P}<0.001)$ compared with the good outcome group (Table 2).

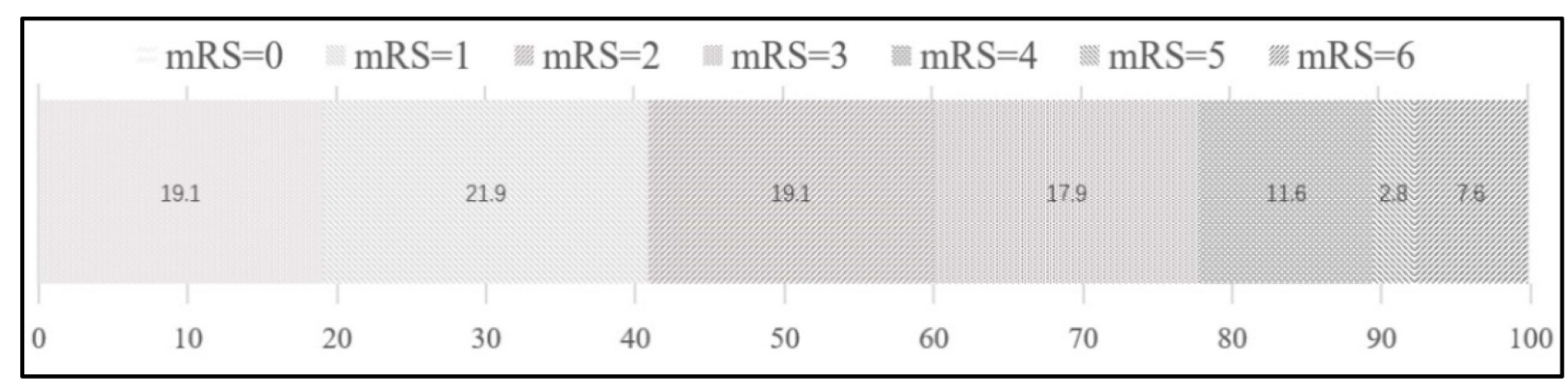

Figure I Distribution of outcomes based on the percentage of the modified Rankin Scale at 3 months. 
Table 2 Comparison of Baseline and Clinical Data Between Two Outcome Groups

\begin{tabular}{|c|c|c|c|}
\hline \multirow[t]{2}{*}{ Variable } & $\begin{array}{c}\text { Poor } \\
\text { Outcome }\end{array}$ & $\begin{array}{c}\text { Good } \\
\text { Outcome }\end{array}$ & \multirow[t]{2}{*}{$P$ value } \\
\hline & $(n=100)$ & $(n=|5|)$ & \\
\hline Age, years, median (IQR) & $\begin{array}{l}75(70, \\
78.75)\end{array}$ & $72(66,75)$ & $<0.001$ \\
\hline Male, n (\%) & $58(58.0)$ & $80(53.0)$ & 0.434 \\
\hline Smoking, n (\%) & $24(24.0)$ & $38(25.2)$ & 0.834 \\
\hline Hypertension, n (\%) & $83(83.0)$ & II $3(74.8)$ & 0.126 \\
\hline Diabetes mellitus, $\mathrm{n}(\%)$ & $23(23.0)$ & $29(19.2)$ & 0.468 \\
\hline Hyperlipidemia, n (\%) & $14(14.0)$ & $29(19.2)$ & 0.284 \\
\hline $\begin{array}{l}\text { Congestive heart failure, } \\
\mathrm{n}(\%)\end{array}$ & $30(30.0)$ & $22(14.6)$ & 0.003 \\
\hline $\begin{array}{l}\text { Baseline NIHSS score, } \\
\text { median (IQR) }\end{array}$ & $15(11,19)$ & $4(2,7)$ & $<0.001$ \\
\hline $\begin{array}{l}\text { Moderate to severe LA, } \\
\mathrm{n}(\%)\end{array}$ & $39(39.0)$ & $24(15.9)$ & $<0.001$ \\
\hline sICH, n (\%) & $17(17.0)$ & $10(6.6)$ & 0.009 \\
\hline nsICH, n (\%) & $30(30.0)$ & $16(10.6)$ & $<0.001$ \\
\hline $\begin{array}{l}\text { Stroke-associated } \\
\text { pneumonia, n (\%) }\end{array}$ & $66(66.0)$ & $13(8.6)$ & $<0.001$ \\
\hline $\begin{array}{l}\text { Anticoagulant therapy, } \\
\text { n (\%) }\end{array}$ & $56(56.0)$ & $64(42.4)$ & 0.881 \\
\hline Stroke recurrence, n (\%) & $4(4.0)$ & $4(2.6)$ & 0.953 \\
\hline \multicolumn{4}{|c|}{ Laboratory findings, $\mathrm{mmol} / \mathrm{L}$ (mean $\pm \mathrm{SD}$ ) } \\
\hline Total cholesterol & $4.1 \pm 0.9$ & $4.1 \pm 0.8$ & 0.482 \\
\hline Triglyceride & $1.0 \pm 0.4$ & $1.3 \pm 0.9$ & 0.005 \\
\hline LDL-C & $2.3 \pm 0.7$ & $2.2 \pm 0.7$ & 0.152 \\
\hline Fasting glucose & $7.0 \pm 2.7$ & $5.8 \pm 1.7$ & $<0.001$ \\
\hline
\end{tabular}

\section{Logistic Regression Analysis}

To further evaluate the independent risk factors for the outcome in CES patients, binary logistic regression analysis was performed. As shown in Table 3, after adjusting for variables with $P<0.2$ in the univariate analysis, the result indicated that the baseline NIHSS score (OR $=1.365,95 \%$ $\mathrm{CI}=1.235-1.509, \mathrm{P}<0.001)$, moderate to severe LA (OR $=3.987,95 \% \mathrm{CI}=1.376-11.551, \mathrm{P}=0.011), \mathrm{nsICH}(\mathrm{OR}$ $=3.924,95 \% \mathrm{CI}=1.254-12.279, \mathrm{P}=0.019), \mathrm{SAP}(\mathrm{OR}=$ $5.392,95 \% \mathrm{CI}=2.012-14.453, \mathrm{P}=0.001)$, and fasting glucose $(\mathrm{OR}=1.279,95 \% \mathrm{CI}=1.012-1.617, \mathrm{P}=0.040)$ were independent risk factors for a poor functional outcome at 3 months.

\section{Discussion}

In this study, we found that the baseline NIHSS score, moderate to severe LA, nsICH, SAP, and fasting glucose were the main risk factors for a poor functional outcome at 3 months after CES onset. Thus, we provided five reliable variables for predicting a poor outcome at 3- months after CES that are based on routinely available data.

Previous studies have found that the CES infarct volume is relatively large, which can lead to a more severe neurological deficit, higher risk of malignant edema and hemorrhagic transformation. ${ }^{15-17}$ Additionally, CES patients tended to be older. ${ }^{18}$ In the current study, the median NIHSS score was 7 (IQR 3-14) and the median age was 73 (IQR 67-77) years, both of which were consistent with results from previous studies. For the prognosis distribution, Hong et al showed that $53 \%$ of patients with CES had a poor prognosis (mRS $\geq 2$ ) at 3 months. ${ }^{4}$ This proportion was $41 \%$ in another registry study. ${ }^{3}$ Our study shows similar results, suggesting that the prognosis of patients with CES is relatively poor.

The NIHSS score, which represents the degree of neurological deficit, is widely used to assess the stroke severity. Studies have shown that the NIHSS score had ideal specificity, sensitivity, and accuracy to predict the prognosis of patients with ischemic stroke. ${ }^{19,20}$ Our findings further confirmed the baseline NIHSS score was an independent risk factor for the functional outcome in CES patients. In our study, for each one additional NIHSS score point, the odds of a poor outcome increased by about 1.4 -fold. We also found that patients with a higher NIHSS score have a higher risk of developing SAP, which is consistent with previous research. ${ }^{21}$ We speculate that the possible reasons are the widespread disturbance of consciousness, indwelling gastric cannula, paralysis, and assisted breathing in patients with much more severe neurological deficits. In addition, the NIHSS score is a risk factor for $\mathrm{sICH}$ and $\mathrm{nsICH}$, which can increase the severity of stroke and prolong the hospital stay. ${ }^{22,23}$ Therefore, the baseline neurological deficit is the main determinant of the CES outcome.

SAP is a major complication that affects the outcome of stroke patients. ${ }^{14,23}$ In our study, $66 \%$ of patients with a poor outcome had SAP, compared with $8.6 \%$ in patients with good outcome. Notably, sICH was a predictor for a poor outcome in the univariate analysis but not in the 
Table 3 Binary Logistic Regression Model of Risk Factors for 3-Month Functional Outcome

\begin{tabular}{|l|c|c|c|c|c|c|}
\hline Variable & B & S.E. & Wald & OR & 95\% CI & P value \\
\hline Baseline NIHSS score & 0.311 & 0.051 & 36.960 & 1.365 & $1.235-1.509$ & $<0.001$ \\
Moderate to severe LA & 1.383 & 0.543 & 6.494 & 3.987 & $1.376-11.551$ & 0.011 \\
nsICH & 1.367 & 0.582 & 5.516 & 3.924 & $1.254-12.279$ & 0.019 \\
SAP & 1.685 & 0.503 & 11.221 & 5.392 & $2.012-14.453$ & 0.001 \\
Fasting glucose & 0.246 & 0.120 & 4.232 & 1.279 & $1.012-1.617$ & 0.040 \\
\hline
\end{tabular}

multivariate analysis model, which is probably because patients with a higher NIHSS score have a greater possibility of developing sICH. Our findings, together with results of the clinical studies mentioned above, may suggest that therapies to reduce the functional deficit at admission and prevention of critical complications are more meaningful in the early-stage treatment of CES.

Leukoaraiosis (LA) is an important surrogate marker of aging and cerebral small vessel disease. Previous reports indicated that the LA burden is highest in patients with lacunar stroke. ${ }^{24}$ In our study, there were 63 patients (25.1\%) with moderate to severe LA. Currently, LA is usually thought to be correlated with blood-brain barrier disruption and microcirculation dysfunction. ${ }^{25}$ Thus, it can act as a "background" impact for the stroke outcome. However, previous studies about the effect of LA on the prognosis of CES showed inconsistent results. Leonards et $\mathrm{al}^{26}$ evaluated brain LA using the Fazekas scale, and they found that moderate to severe LA (Fazekas $\geq 3$ ) was related to the 1-year functional disability in patients with mild ischemic stroke. In their study, there was no significant difference in Fazekas scores between patients with the CES stroke subtype and those with atherosclerosis or small vascular disease. However, another study demonstrated that the volume of abnormal white matter in LA was positively correlated with a poor outcome, but this correlation was not significant in patients with $\mathrm{CES} .{ }^{24} \mathrm{~A}$ possible explanation for this phenomenon is that patients with CES usually have larger infarct volumes, and thus, greater functional deficits, which is the critical factor that determines the outcome.

Our study has some limitations. First, this retrospective study was conducted as a single-center database study, and it had a relatively small sample size, which may increase the possibility of selection bias. Second, because the aim was to find practical clinical indicators to predict the prognosis of patients with CES, this study did not accurately quantify the infarct and LA volumes.
In conclusion, we provided clinicians with five reliable and practical clinical indicators that may predict the outcome of patients with acute CES. Although this is a retrospective study with a relatively small sample size, we believe that our data conveys important insights.

\section{Abbreviations}

CES, cardioembolic stroke; CI, confidence intervals; CT, computed tomography; CTA, computed tomography angiography; ECG, electrocardiogram; ICH, intracranial hemorrhage; IQR, interquartile range; LA, leukoaraiosis; MRA, magnetic resonance angiography; MRI, magnetic resonance imaging; mRS, modified Rankin Scale; NIHSS, National Institutes of Health Stroke Scale; nsICH, nonsymptomatic intracranial hemorrhage; OR, odds ratio; SAP, stroke-associated pneumonia; sICH, symptomatic intracranial hemorrhage.

\section{Data Sharing Statement}

The datasets during and/or analyzed during the current study available from the corresponding author on reasonable request.

\section{Ethics Approval and Informed Consent}

This study was approved by the Clinical Research Ethics Committee of the Affiliated Changzhou No.2 People's Hospital of Nanjing Medical University (2018KY03201). A written informed consent was obtained from the patients or their legally authorized representatives. This study was conducted in accordance with the Declaration of Helsinki.

\section{Consent for Publication}

A written informed consent for publication was obtained from the patients or their legally authorized representatives. All patient data were de-identified. 


\section{Funding}

There is no funding to report.

\section{Disclosure}

The authors declare that there is no conflict of interest.

\section{References}

1. Feigin VL, Nichols E, Alam T. Global, regional, and national burden of neurological disorders, 1990-2016: a systematic analysis for the Global Burden of Disease Study 2016. Lancet Neurol. 2019;18 (5):459-480. doi:10.1016/S1474-4422(18)30499-X

2. Celeste F, Muratori M, Mapelli M, et al. The evolving role and use of echocardiography in the evaluation of cardiac source of embolism. $J$ Cardiovasc Echogr. 2017;27(2):33-44. doi:10.4103/jcecho. jcecho_1_17

3. Tan YF, Zhan LX, Chen XH, et al. Risk factors, clinical features and prognosis for subtypes of ischemic stroke in a Chinese population. Curr Med Sci. 2018;38(2):296-303. doi:10.1007/s11596-018-1878-1

4. Hong KS, Lee J, Bae HJ, et al. Greater stroke severity predominates over all other factors for the worse outcome of cardioembolic stroke. J Stroke Cerebrovasc Dis. 2013;22(8):e373-e380. doi:10.1016/j. jstrokecerebrovasdis.2013.04.008

5. Li L, Yiin GS, Geraghty OC, et al. Incidence, outcome, risk factors, and long-term prognosis of cryptogenic transient ischaemic attack and ischaemic stroke: a population-based study. Lancet Neurol. 2015;14(9):903-913. doi:10.1016/S1474-4422(15)00132-5

6. Arauz A, Ruiz-Navarro F, Barboza MA, et al. Outcome, recurrence and mortality after non-valvular atrial fibrillation stroke: long-term follow-up study. J Vasc Interv Neurol. 2017;9(6):5-11.

7. Goyal M, Menon BK, van Zwam WH, et al. Endovascular thrombectomy after large-vessel ischaemic stroke: a meta-analysis of individual patient data from five randomised trials. Lancet. 2016;387 (10029):1723-1731. doi:10.1016/S0140-6736(16)00163-X

8. Zhao W, Xu J, Li S, et al. Low-dose tirofiban is associated with reduced in-hospital mortality in cardioembolic stroke patients treated with endovascular thrombectomy. J Neurol Sci. 2021;427:117539. doi:10.1016/j.jns.2021.117539

9. Jeong HY, Jung KH, Mo H, et al. Characteristics and management of stroke in Korea: 2014-2018 data from Korean Stroke Registry. Int J Stroke. 2020;15(6):619-626. doi:10.1177/1747493019884517

10. Leng T, Xiong ZG. Treatment for ischemic stroke: from thrombolysis to thrombectomy and remaining challenges. Brain Circ. 2019;5 (1):8-11. doi:10.4103/bc.bc_36_18

11. Adams HP, Bendixen BH, Kappelle LJ, et al. Classification of subtype of acute ischemic stroke. Definitions for use in a multicenter clinical trial. TOAST. Trial of Org 10172 in acute stroke treatment. Stroke. 1993;24(1):35-41. doi:10.1161/01.STR.24.1.35

12. Wahlund LO, Barkhof F, Fazekas F, et al. A new rating scale for age-related white matter changes applicable to MRI and CT. Stroke. 2001;32(6):1318-1322. doi:10.1161/01.STR.32.6.1318

13. Larrue V, von Kummer RR, Müller A, et al. Risk factors for severe hemorrhagic transformation in ischemic stroke patients treated with recombinant tissue plasminogen activator: a secondary analysis of the European-Australasian Acute Stroke Study (ECASS II). Stroke. 2001;32(2):438-441. doi:10.1161/01.STR.32.2.438
14. de Montmollin E, Ruckly S, Schwebel C, et al. Pneumonia in acute ischemic stroke patients requiring invasive ventilation: impact on short and long-term outcomes. J Infect. 2019;79(3):220-227. doi:10.1016/j.jinf.2019.06.012

15. Jung JM, Kwon SU, Lee JH, et al. Difference in infarct volume and patterns between cardioembolism and internal carotid artery disease: focus on the degree of cardioembolic risk and carotid stenosis. Cerebrovasc Dis. 2010;29(5):490-496. doi:10.1159/000297965

16. Wu S, Yuan R, Wang Y, et al. Early prediction of malignant brain edema after ischemic stroke. Stroke. 2018;49(12):2918-2927. doi:10.1161/STROKEAHA.118.022001

17. Gill D, Baheerathan A, Aravind A, et al. Severe hemorrhagic transformation after thrombolysis for acute ischemic stroke prevents early neurological improvement. J Stroke Cerebrovasc Dis. 2016;25 (9):2232-2236. doi:10.1016/j.jstrokecerebrovasdis.2016.04.020

18. Yasuda K, Fukuda S, Nakamura M, et al. Predictors of cardioembolic stroke in Japanese patients with atrial fibrillation in the Fushimi AF registry. Cerebrovasc Dis Extra. 2018;8(2):50-59. doi:10.1159/ 000488206

19. Kwakkel G, Veerbeek JM, van Wegen EE, et al. Predictive value of the NIHSS for ADL outcome after ischemic hemispheric stroke: does timing of early assessment matter? J Neurol Sci. 2010;294(12):57-61. doi:10.1016/j.jns.2010.04.004

20. Tai MLS, Goh KJ, Kadir KAA, et al. Predictors of functional outcome in patients with stroke thrombolysis in a tertiary hospital in Malaysia. Singapore Med J. 2019;60(5):236-240. doi:10.11622/ smedj.2018150

21. Huang GQ, Lin YT, Wu YM, et al. Individualized prediction of stroke-associated pneumonia for patients with acute ischemic stroke. Clin Interv Aging. 2019;14:1951-1962. doi:10.2147/CIA. S225039

22. Yaghi S, Willey JZ, Cucchiara B, et al. Treatment and outcome of hemorrhagic transformation after intravenous alteplase in acute ischemic stroke: a scientific statement for healthcare professionals from the American Heart Association/American Stroke Association. Stroke. 2017;48(12):e343-e361. doi:10.1161/STR.0000000000000152

23. Parikh NS, Merkler AE, Iadecola C. Inflammation, autoimmunity, infection, and stroke: epidemiology and lessons from therapeutic intervention. Stroke. 2020;51(3):711-718. doi:10.1161/ STROKEAHA.119.024157

24. Ryu WS, Woo SH, Schellingerhout D, et al. Stroke outcomes are worse with larger leukoaraiosis volumes. Brain. 2017;140 (1):158-170. doi:10.1093/brain/aww259

25. Wardlaw JM, Smith C, Dichgans M. Small vessel disease: mechanisms and clinical implications. Lancet Neurol. 2019;18(7):684-696. doi:10.1016/S1474-4422(19)30079-1

26. Leonards CO, Ipsen N, Malzahn U, et al. White matter lesion severity in mild acute ischemic stroke patients and functional outcome after 1 year. Stroke. 2012;43(11):3046-3051. doi:10.1161/STROKEAHA.111.646554 


\section{Publish your work in this journal}

Neuropsychiatric Disease and Treatment is an international, peerreviewed journal of clinical therapeutics and pharmacology focusing on concise rapid reporting of clinical or pre-clinical studies on a range of neuropsychiatric and neurological disorders. This journal is indexed on PubMed Central, the 'PsycINFO' database and CAS, and is the official journal of The International Neuropsychiatric Association (INA). The manuscript management system is completely online and includes a very quick and fair peer-review system, which is all easy to use. Visit http://www.dovepress.com/testimonials.php to read real quotes from published authors.

Submit your manuscript here: https://www.dovepress.com/neuropsychiatric-disease-and-treatment-journal 